\title{
EFFECTS OF PERTURBATIONS ON ACCURACY OF ARM MOVEMENTS
}

\author{
JEROME N. SANES ${ }^{1}$ AND EDWARD V. EVAR'TS \\ Laboratory of Neurophysiology, National Institute of Mental Health, Bethesda, Maryland 20205
}

Received February 2, 1982; Revised December 6, 1982; Accepted December 22, 1982

\begin{abstract}
This study was carried out to test the hypothesis that the magnitude of perturbation-induced disruption of active movement depends upon the size of the active movement, with small movements being more disrupted than large ones. Human subjects performed pronating or supinating arm movements of $3^{\circ}, 10^{\circ}$, and $30^{\circ}$ in a discrete visual tracking task. When the movements were briefly stopped or reversed, the resultant errors in trajectory and static position (expressed relative to the movement size) were greater for small as compared to large movements. These findings demonstrate that peripheral kinesthetic disturbances modify movement trajectory and end point error, with small movements being more affected than large movements.
\end{abstract}

Despite intensive investigation, the nature of the regulatory role of proprioceptive information in movement control has remained uncertain. Whereas it is quite clear that some kinesthetic input is required to identify and maintain static limb position, the importance of information derived from these same peripheral receptors in voluntary movement has been challenged. Lashley (1917) was perhaps the first to report the retained ability of a patient to move a deafferented extremity with reasonable accuracy. After this early demonstration, the results of numerous investigations have been interpreted to diminish the role of peripheral input while elevating that of central neural commands in controlling the accuracy of active movements. For example, several recent studies have shown a lack of major deficits in moving the head or a limb to a prelearned position after surgical deafferentation in monkeys or ischemic deafferentation in humans (Bizzi et al., 1976, 1978; Polit and Bizzi, 1979; Kelso and Holt, 1980). In addition, the kinematics of a variety of movements have been found to be relatively invariant (Bernstein, 1967; Terzuolo and Vivani, 1980; Soechting and Lacquaniti, 1981), suggesting that central motor commands entirely control those movements. There is, however, other evidence that demonstrates the importance of afferent input in a variety of motor tasks performed by humans. Altered position sense and sense of effort (Goodwin et al., 1972; Gandevia and McCloskey, $1977 \mathrm{a}, \mathrm{b})$ and disruption of fine motor tasks, such as reproducing alphabetic characters (Laszlo and Bairstow, 1971), were observed when a subject's limb was deaffer-

${ }^{1}$ To whom correspondence should be addressed at Laboratory of Neurophysiology, National Institute of Mental Health, Building 36, Room 2D-10, Bethesda, MD 20205. ented by ischemia. It has also been suggested that acute inactivation of the gamma loop in humans impairs the ability to control motor unit discharge (Fukushima et al., 1976).

A number of the previous studies aimed at elucidating the role of proprioceptive inputs in voluntary movement have involved the use of relatively large mechanical disturbances imposed in the course of relatively large movements (Polit and Bizzi, 1979; Kelso and Holt, 1980; Schmidt and McGown, 1980). However, there is evidence that cutaneous and muscle receptors have nonlinear response profiles with the greatest sensitivity for small amplitude signals (Matthews and Stein, 1969; Poppele and Bowman, 1970; Hasan and Houk, 1975; Knibestöl, 1975; Goodwin et al., 1978; Knibestöl and Vallbo, 1980), thereby suggesting that the contribution of peripheral receptors in active movement might be more clearly determined if fine movements were studied. The present experiments were undertaken to determine whether peripheral disturbances delivered to the arm caused greater impairments of small as compared to large voluntary movements.

\section{Materials and Methods}

In the first experiment 11 subjects ( 8 females, 3 males) between the ages of 21 and 35 years and without neurological abnormalities were studied. Nine of the subjects were naive with respect to the design and goals of the experiment. Subjects grasped a handle that could be rotated by forearm pronation or supination while the forearm was secured between two metal supports. The grasped handle was coupled to the axle of a low friction brushless DC torque motor (Aeroflex TQ64W-5). Each 
subject viewed two vertically oriented oscilloscope beams on a large display Wavetek oscilloscope (model 1901B). One beam represented a target and the second beam corresponded to the orientation of the handle. Subjects were instructed to maintain alignment between the two beams and to reestablish alignment when the target beam jumped to a new position. The magnitude of handle rotation necessary to establish realignment was $30^{\circ}$ in certain blocks of trials, $10^{\circ}$ in other blocks of trials, and $3^{\circ}$ in still other blocks of trials. The distance on the video screen between the hold zone and the final target was constant, but the amount of angular displacement necessary to realign the oscilloscope beams varied according to movement size. For all trials, the target beam remained displaced from the center of the display until subjects moved the handle to reestablish and then maintain realignment for 1.2 to $2.0 \mathrm{sec}$ in a small zone ( $\pm 5 \%$ of the movement size) surrounding the target. The hold period was begun anew if a subject failed to hold the handle within the initial window for the allotted time period. At the end of the hold period, the target beam jumped to the center of the video tube. Subjects were instructed to move the handle as quickly and as accurately as possible to match the handle (position) beam with the target beam. The final position for all movements was $0^{\circ}$ of forearm rotation (i.e., vertical orientation of the handle).

Subjects completed three sessions: first, a practice, and then two test sessions. Across sessions, the blocks of each movement size were performed in counterbalanced order. In the practice session and separately for each movement amplitude, subjects first performed 150 pronation or supination movements of the forearm when the oscilloscope beam corresponding to handle position was always available to guide the limb's movement and end point positioning. Improvements in accuracy and increases in movement speed were encouraged until subjects usually (greater than $70 \%$ of the movements) performed the initial phases of the movement within $250 \mathrm{msec}$ to an accuracy of $\pm 15 \%$. For an additional 100 practice trials the handle position beam was pseudorandomly blanked on $50 \%$ of the trials when the handle was moved by the subject outside of the initial hold zone. Similar to movement performance with visual guidance, when handle position was not displayed subjects were instructed to move rapidly and accurately toward the target and to stop moving and maintain a static forearm posture as soon as possible after the initial phases of movement.

After practice, two test sessions were conducted on two subsequent and separate occasions during which subjects separately performed 250 test trials of each

TABLE I

Trial type and probability of occurrence during the test session

\begin{tabular}{cclc}
\hline Direction & Visual Guidance & Perturbation $^{a}$ & Probability \\
\hline Pronation & Absent & Absent & 0.208 \\
& & Present & 0.042 \\
& Present & Absent & 0.208 \\
& & Present & 0.042 \\
Supination & Absent & Absent & 0.208 \\
& & Present & 0.042 \\
& Present & Absent & 0.208 \\
& & Present & 0.042 \\
\hline
\end{tabular}

${ }^{a}$ Perturbation was either a stop or a reversal of movement.

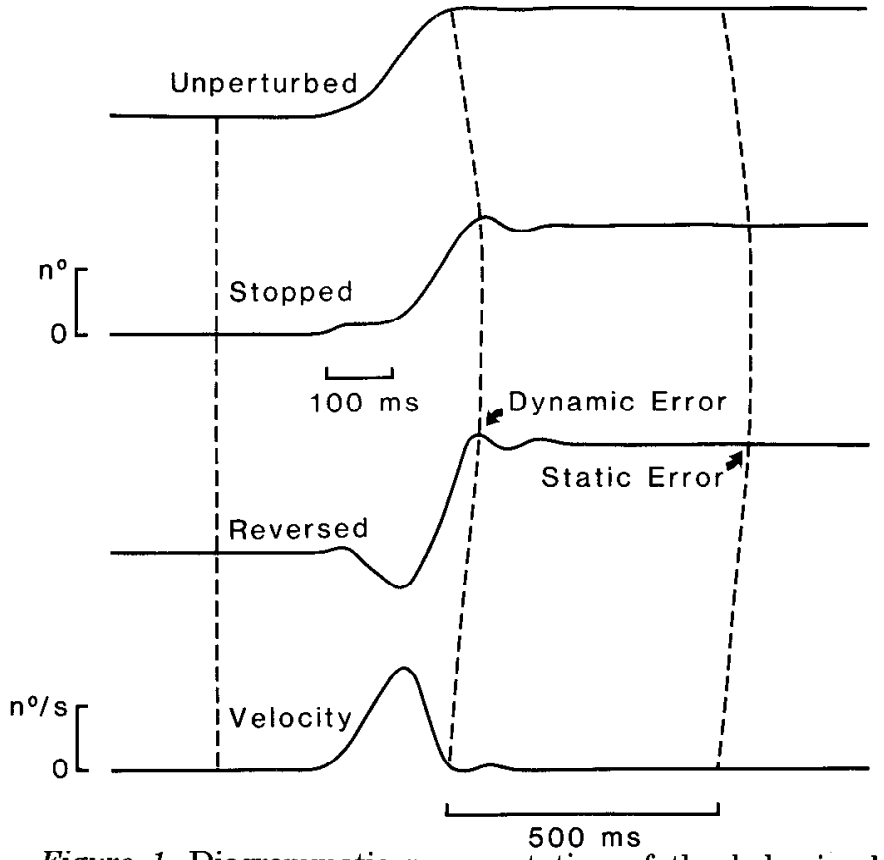

Figure 1. Diagrammatic representation of the behavioral situation. The upper three traces schematically illustrate position of the torque motor handle for an unobstructed, a stopped, and reversed supination movement, respectively. Note that in the second and third trace the handle movement is obstructed for $100 \mathrm{msec}$ nearly immediately after the onset of movement. The bottom trace represents handle velocity for an unobstructed movement. The leftmost vertical dashed line indicates when the target beam jumped to the middle of the display screen (i.e., the cue requesting the subject to begin a rapid movement to realign the handle position with the target beam). The other vertical dashed lines indicate when dynamic and static errors in performance were measured (see the text for details). The vertical scales to the left represent angular displacement of the handle (degrees) and handle velocity (degrees per second). The calibration bars are ambiguously scaled since the studied movements were scaled in the ratio of 10:3:1 (for $3^{\circ}, 10^{\circ}$, and $30^{\circ}$, respectively).

movement size $\left(3^{\circ}, 10^{\circ}\right.$, and $\left.30^{\circ}\right)$. In these sessions the arm movements were either sometimes briefly reversed or stopped. The passive mechanical stimuli were produced by switching, for $100 \mathrm{msec}$, the torque motor from a torque commanded to a position-feedback mode. The electronic circuitry that controlled the motor received no external signal to produce the stop stimulus, but a voltage ramp was provided to produce the reversal of limb motion. When movement size was changed, subjects were given sufficient practice (typically 5 to 50 trials). Table I shows the probabilities of trial types that were pseudorandomly presented. If the forearm was obstructed, the perturbation began when the handle was moved beyond the initial hold zone. The stop stimulus lasted for $100 \mathrm{msec}$ and when movement was reversed the forearm was moved backwards one-third of the intended movement magnitude in $100 \mathrm{msec}$. Although the perturbations were novel to the subject in the test session, initial startle responses or habituation of error across trials were not observed.

Figure 1 illustrates the behavioral situation and the general scheme for data analysis. Movement errors were measured when velocity first reached zero (dynamic er- 
ror) and from 500 to $550 \mathrm{msec}$ after this point (static error). Absolute and relative errors in performance were computed for both dynamic and static errors. Absolute error was the angular difference between the actual handle position and the vertical handle position called for by the target, whereas relative error was expressed in relation to each movement size. For non-perturbation trials, movement time (MT) was measured within $5 \mathrm{msec}$ accuracy from the first change in velocity after the target jumped to the center of the video tube to the first zerocross of velocity. On perturbation trials, MT was measured by summing the time between movement and perturbation onset plus the time from the end of the mechanical disturbance to the first zero-cross in velocity. The MT analysis for the stop experiment was based on data obtained from nine subjects. MT information was available from all 11 subjects for the experiment where forearm rotation was periodically reversed. Dynamic, static, and variable (standard deviation about the positional deviations) errors and MTs were averaged across conditions and analyzed for significance with a repeated measures analysis of variance.

In a second experiment additional properties of the forearm were examined. The subjects (five males, ages 27 to 32 years) were required to maintain a half-pronated position of the forearm by aligning two oscilloscope beams as described above. After alignment was established and held for 2 to $5 \mathrm{sec}$, the handle was moved either $0.33^{\circ}, 1^{\circ}, 3.33^{\circ}$, or $10^{\circ}$ in a smooth ramp that lasted 100 msec. The ramp stimuli were generated by switching, for $100 \mathrm{msec}$, the motor from a torque commanded to a position-feedback mode and then providing a variably sized $100-$ msec voltage ramp to the control circuitry of the torque motor. One ramp size was repeated for 50 trials; on one-half of the trials the forearm was pseudorandomly passively supinated, on the remaining trials the forearm was pronated. The oscilloscope beam that indicated handle position was blanked at ramp onset and reappeared $2 \mathrm{sec}$ later. Subjects were instructed not to interfere with the effects of the ramp until the oscilloscope beam corresponding to handle position reappeared. On each trial the deviation (in degrees) from the initial holding position was measured and averaged from 500 to $550 \mathrm{msec}$ (at 5 -msec intervals) after the ramp stimulus had ceased. These data were segregated according to displacement size and movement direction and were either summed irrespective of the direction of final position (signed error) - positive error was in the direction of the displacement and negative error was overshoot in the direction opposite to the displacement-or summed by adding the absolute values of each deviation from the original position (absolute error). These values were then expressed as a percentage of the displacement size.

\section{Results}

The effects of briefly reversing or stopping the forearm were qualitatively and quantitatively similar and therefore will be considered together.

\footnotetext{
${ }^{2}$ The $F$ values and degrees of freedom will not be presented under "Results" but are available on request from the authors. Except where noted, the probability level for a particular significant effect refers to both the stop and reverse experiment.
}

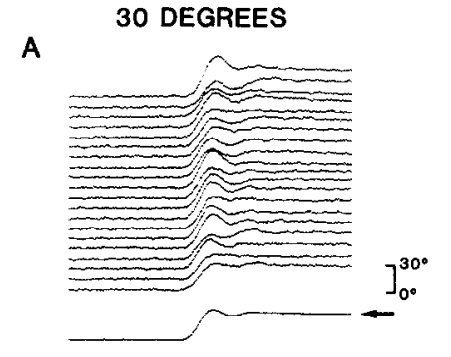

3 DEGREES

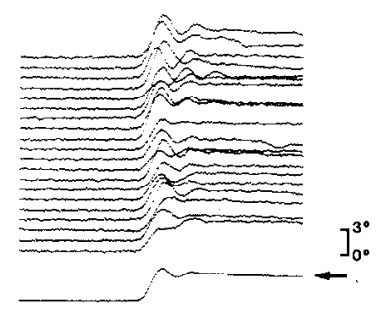

B
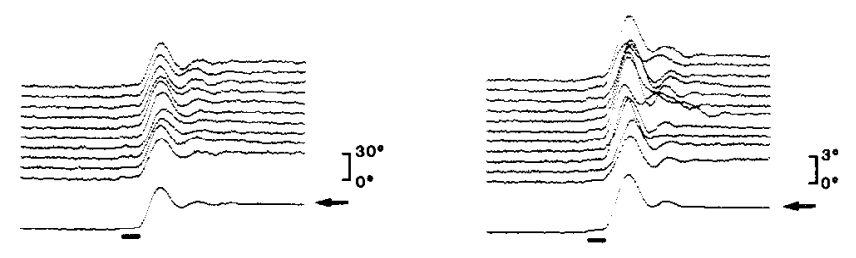

C
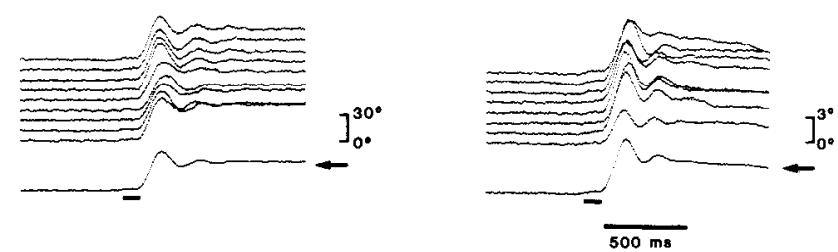

Figure 2. Unobstructed and stopped supination movements from subject $S G$. $A$, Individual trials and average responses for forearm supinations of $30^{\circ}$ (left) and $3^{\circ}$ (right) when movements were unperturbed and performed without visual guidance. There was more variability in the trajectory and end point control for smaller voluntary movements and more dynamic overshoot for the $3^{\circ}$ movement (compare average traces of the two movement sizes). The arrows to the right of the average responses indicate intended final position. $B$, Forearm supination with visual guidance when voluntary movement was briefly slopped. The bar underneath the averaged response indicates when the movement was stopped. The variance and relative error for the trajectory were greater for the small movement. An accurate final position was easily attained for both movement sizes since visual guidance was available. $C$, Large and small obstructed movements performed without visual guidance. The relative trajectory and static error and variability in error were greater for small movements.

Error in forearm trajectory. The forearm trajectory of subject SG's unopposed and stopped supination movements of $30^{\circ}$ and $3^{\circ}$ are illustrated in Figure 2. In this figure the $3^{\circ}$ and $30^{\circ}$ movements have been scaled to make the figures comparable in relative error. For this subject, dynamic error was scaled with respect to movement size when unobstructed movements* were performed (Fig. 2A). When SG's forearm movements were briefly stopped, the relative dynamic error increased for both movement sizes that are illustrated (Fig. 2B), though enhancement of relative dynamic error was greatest for the smallest $\left(3^{\circ}\right)$ movement. ${ }^{3}$

\footnotetext{
${ }^{3}$ Our analysis of kinematic data used relative error instead of absolute error (expressed in degrees of inaccuracy). The rationale for this choice was our belief that accuracy should be considered in the context of the control signal for a movement. A simple example of two types of goal-directed movements will illustrate this belief. Whereas slight absolute errors in displacement are not that significant for large reaching
} 
STOP DYNAMIC

$A$ SUPINATION
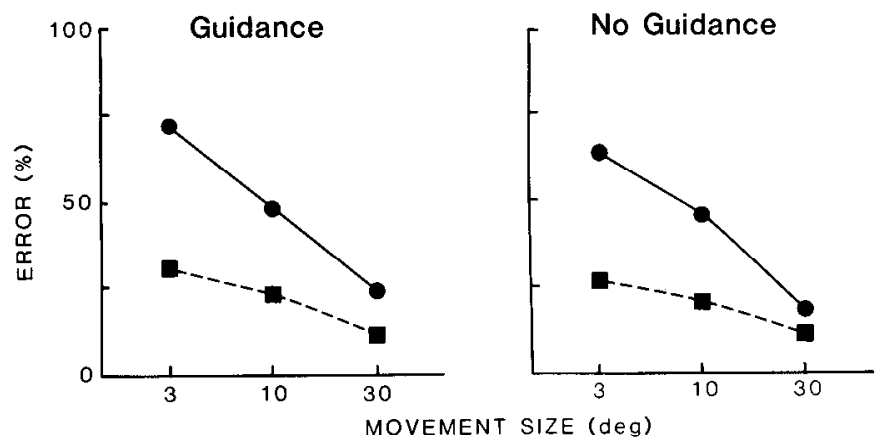

B

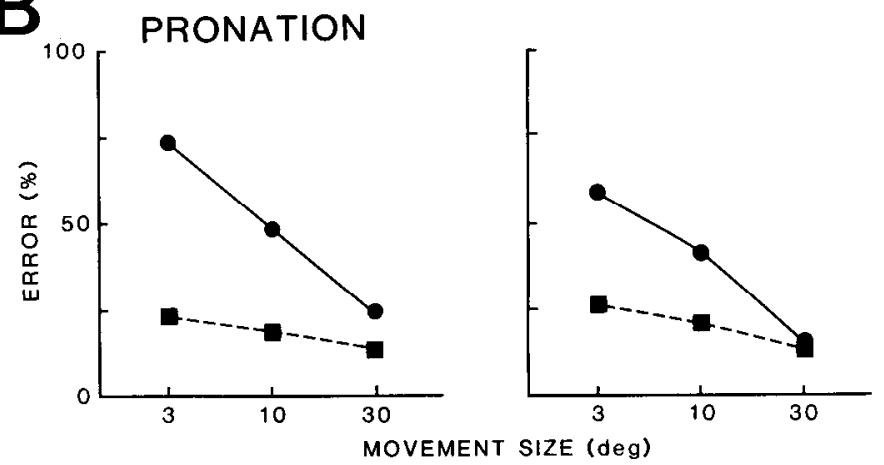

Figure 3. Dynamic error for the group of subjects when movement was stopped. As noted in the text, relative dynamic error (ordinate) increased as the movement size decreased (abscissa). This was evident for both supination $(A)$ and pronation $(B)$ movements, when the voluntary handle movement was unobstructed (dashed lines) or briefly stopped (solid lines), and when subjects had visual guidance of the handle position (Guidance) or were unable to see the orientation of the handle position (No Guidance). When the handle movement was briefly stopped, note that the relative dynamic error increased a greater amount for the small $\left(3^{\circ}\right)$ in comparison to the large $\left(30^{\circ}\right)$ movements. This effect was evident for all movement conditions.

The grouped and most individual data for experiments in which the forearm was briefly stopped (Fig. 3) or reversed (Fig. 4) were similar to the observations seen for subject SG. The most significant observation for the group of subjects was the inverse relationship between relative dynamic error and movement amplitude in both the stop and reverse experiments $(p<0.001)$. The increase in dynamic error was also linear $(p \leq 0.0001)$. Figures 3 and 4 also illustrate the effects that stopping or reversing, respectively, the forearm movements had on the forearm trajectory (compare dashed lines, no-perturbation, with solid lines, perturbation, Figs. 3 and 4). When movements were obstructed, the relative dynamic error increased $(p \leq 0.0005)$. Furthermore, there was a

movements, the same magnitude of error would be detrimental to the manual control of ballistic aiming devices or of surgical instruments. It should be pointed out that, contrary to the results derived from measurement of relative error, the absolute dynamic and static positioning and variable errors were directly related to movement size.
REVERSE DYNAMIC

\section{A supination}
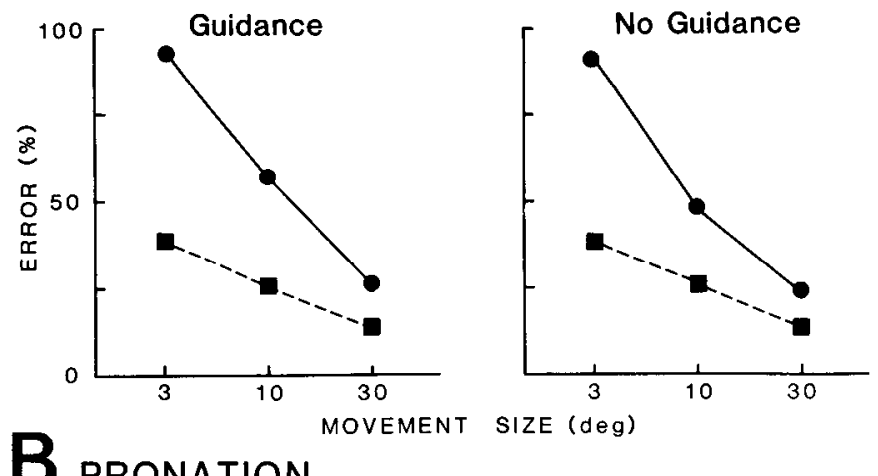

PRONATION
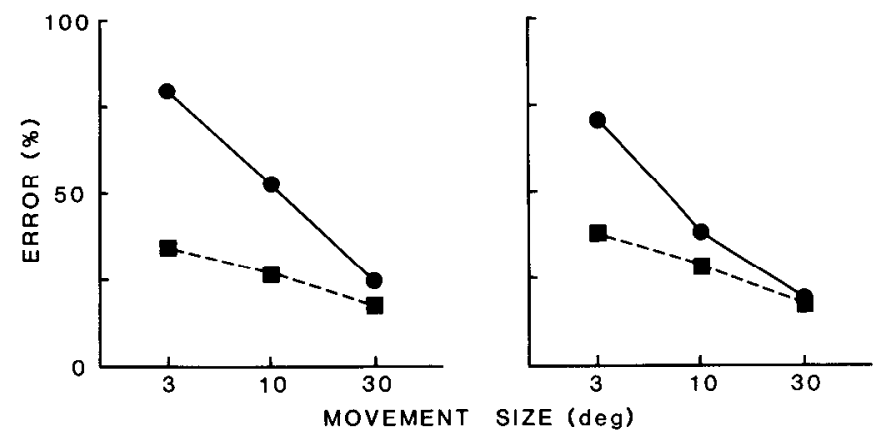

Figure 4. Dynamic error when movements were reversed. Relative dynamic error for the group of subjects when the handle was moved by the motor in the direction opposite from the intended movement. Similar to the results with the stop stimulus, the reversal ramp caused dynamic error to be inversely related to movement size. The error also increased more when $3^{\circ}$ movements were perturbed in comparison to $30^{\circ}$ movements. All symbols and description are as in Figure 3.

substantially greater increase in dynamic error for the small in comparison to the large movements following brief obstructions of forearm movements ( $p \leq 0.0001$ ). Indeed, it was noted that perturbations hardly affected relative error when subjects performed the largest $\left(30^{\circ}\right)$ movement. Somewhat surprisingly, dynamic error was smaller when visual guidance of the handle position was absent $(p<0.05)$. The reliability of the main effect of visual guidance became apparent when comparing the dynamic error when perturbations were delivered to the arm while subjects operated under the two conditions of visual guidance (guidance-perturbation interaction; $p \leq$ 0.05). Thus, dynamic error was smaller when subjects lacked visual guidance while the forearm was perturbed. There was no difference in dynamic error for movements in different directions $(p>0.05)$.

Variability in dynamic positional error. The variability in forearm trajectory for the group of subjects is illustrated in Figure 5. This measure was derived by summing together the individual standard deviations of positional error. Similar to the observations on positional error, the variation in relative dynamic error increased as movement size decreased ( $p \leq 0.0001)$. The linear and quadratic components of the function relating variable dynamic error and movement size were reliable in both 

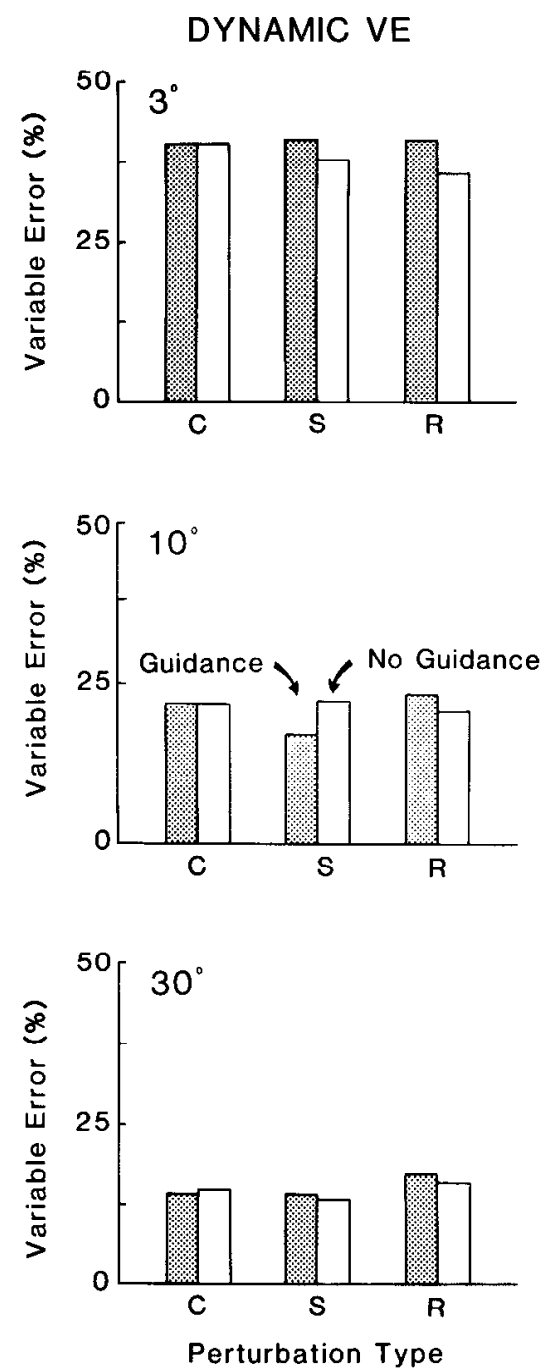

Figure 5. Dynamic variable error (VE). Variability in trajectory of both directions of forearm rotation (summed together) for unobstructed control $(C)$, stopped $(S)$, and reversed $(R)$ movements of $3^{\circ}, 10^{\circ}$, and $30^{\circ}$ amplitude (top to bottom) expressed as a percentage of the requested movement size. The presence or absence of visual guidance is indicated by hatched bars or open bars, respectively. The values for the control trials were derived by summing together and averaging the values from all trials in which voluntary handle movement was unobstructed. Note that relative variability in the forearm trajectory increases as the movement size decreases, but that perturbations and conditions of visual guidance fail to affect the variation in forearm trajectory.

the stop and reverse experiments $(p \leq 0.05)$. Neither the presence nor the absence of mechanical disturbances or visual guidance affected the variation in relative dynamic error.

Static error. The relative end point deviations of forearm position from the target location for the group data are displayed in Figure 6 for the stopped $(S)$, reversed $(R)$, and nonperturbed trials $(C)$. In the absence of visual guidance of the handle's position, subjects depended on either precise central commands that specified the final state of forearm supinator and pronator muscles or proprioceptive cues that continuously modified neural signals to accurately position the forearm. When visually and nonvisually guided movements were included in the analysis of variance, it was found that relative static error increased as movement size decreased $(p \leq 0.005)$. The linear component for the movement size function was reliable for both experiments $(p \leq 0.005)$. The increase in static error observed when movement size decreased was only apparent for movements performed without visual guidance (size-guidance interaction; $p \leq 0.0001$ ).

The experimental result most relevant to the question whether the arm movements studied were prepro-

\section{STATIC ERROR}
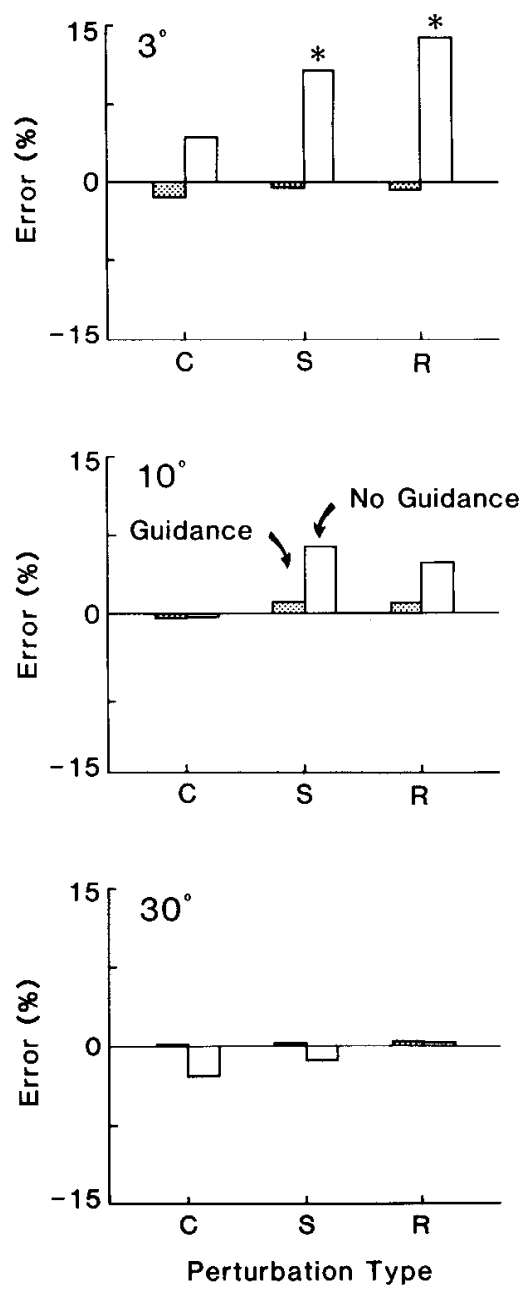

Figure 6. Static positional error. End point error for unobstructed $(C)$, stopped $(S)$, and reversed $(R)$ movements in both directions (summed together) of forearm rotation for the group of subjects. Guidance and No Guidance refer to the visual conditions. Note that when the subjects had visual guidance, end point error of the forearm position was minimal. In the absence of visual guidance and for unobstructed movements, the relative static error was negative, or undershooting the target, for $30^{\circ}$ movements and positive, or overshooting the target, for $3^{\circ}$ movements. End point error became more positive (greater tendency to overshoot) when perturbutions were delivered soon after movement began. Without visual guidance, the greatest deviation of the handle position from that called for by the target was when subjects performed $3^{\circ}$ movements. Aster. isks indicate statistically significant differences $(p<0.05)$ of mean end point error of perturbed compared to unperturbed trials when the visual feedback was held constant. 
grammed was the extent to which perturbations altered end point positioning of the forearm. For both experiments, there was an overall effect of obstructing the movements $(p<0.05)$. Another important issue concerning static limb positioning was whether the perturbation differentially affected small and large movements. The bar graphs in Figure 6 indicate that when subjects performed the $3^{\circ}$ movements, static error was increased when the limb was perturbed and when visual guidance was absent. Table II shows the number of subjects who had end point overshoot and the corresponding mean relative static error for the group. These data illustrate that there was sufficient overlap between end point errors for the three movement sizes; thus the analysis of variance failed to demonstrate a reliable size-perturbation interaction (stop, $p=0.12$; reverse, $p=0.07$ ). A separate analysis of variance based only on the static error obtained when subjects did not have visual guidance revealed reliability of all of the main effects noted above $(p \leq 0.05)$ but still failed to indicate an interaction between movement size and perturbation for relative static error $(p>0.05)$ for the same reasons noted above. When the end point errors of forearm position for two directions of movement were averaged, a reliable interaction term for size and perturbation was found only for the experiment in which the forearm trajectory was periodically reversed ( $p<0.05$; stop experiment, $p=$ 0.16 ). It should be noted that planned comparisons of mean static error revealed that static error was greater when the limb was perturbed in the absence of visual guidance than when the limb was perturbed in the presence of visual guidance and all nonperturbed trials only for the $3^{\circ}$ movement ( $p \leq 0.05$, the Student's $t$ test, two tail). Decreases in movement size or delivery of perturbations did not alter accurate realignment of the handle position when subjects were able to visually monitor handle position.

Variability in static error. The variation in end point error for the group of subjects is shown in Figure 7. When subjects performed smaller movements, the variability in relative static error increased ( $p \leq 0.0001$ ). Both the linear and quadratic components composing the main effect of movement size were significant $(p \leq 0.05)$, suggesting that variability of relative static error first increased and then began to approximate asymptotic

TABLE II

Individual performance of end point positioning

\begin{tabular}{|c|c|c|c|c|}
\hline \multirow{2}{*}{ Movement } & \multicolumn{2}{|r|}{ Pronation } & \multicolumn{2}{|c|}{ Supination } \\
\hline & $N^{a}$ & Mean & $N$ & Mean \\
\hline \multicolumn{5}{|l|}{ Stop stimulus } \\
\hline $3^{\circ}$ & 8 & $9.86(5.48)^{b}$ & 9 & $11.55(4.09)$ \\
\hline $10^{\circ}$ & 11 & $8.86(3.13)$ & 8 & $3.90(4.55)$ \\
\hline $30^{\circ}$ & 5 & $-0.21(3.11)$ & 5 & $-2.57(2.54)$ \\
\hline \multicolumn{5}{|l|}{ Reverse stimulus } \\
\hline $3^{\circ}$ & 7 & $10.12(4.88)$ & 10 & $17.64(6.7)$ \\
\hline $10^{\circ}$ & 7 & $6.12(4.22)$ & 6 & $3.99(2.74)$ \\
\hline $30^{\circ}$ & 6 & $3.04(3.87)$ & 4 & $-2.26(8.8)$ \\
\hline
\end{tabular}

${ }^{a}$ Refers to the number of subjects whose end point error was in the overshoot direction. Maximum $N=11$.

${ }^{b}$ Numbers in parentheses are the SEM.
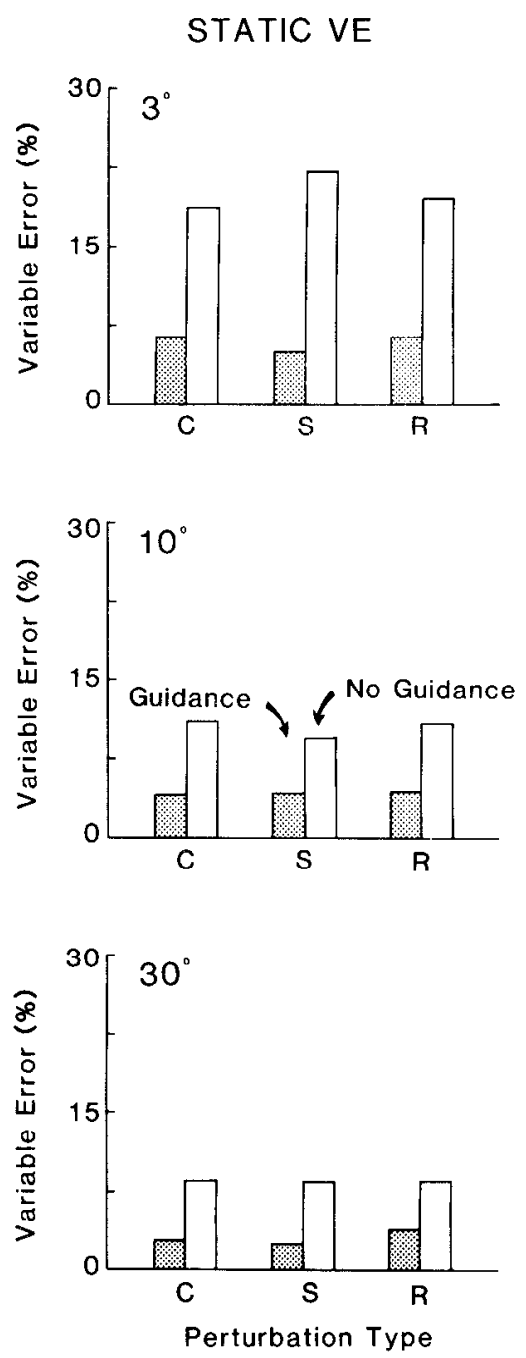

Figure 7. Static variable error (VE). Variation in end point error for the group of subjects. Conditions are as in Figures 5 and 6 . Note that static variable error increases when subjects move without visual guidance and also as the movement size decreases.

values for movements larger than $10^{\circ}$. The variation in relative end point error was greater when subjects performed forearm movements in the absence of visual guidance $(p \leq 0.0001)$. Accurate reproduction of the smaller $3^{\circ}$ inovements was more difficult than the larger $10^{\circ}$ and $30^{\circ}$ movements in the absence of visual guidance (size-guidance interaction, $p \leq 0.0001$ ). Delivery of perturbations did not modify variability of end point positioning of the forearm $(p>0.05)$.

Movement time. The MTs for all of the behavioral conditions are shown in Table III. MT was similar for the three amplitudes of forearm rotation for the stop experiment, but small movements were performed faster in the reverse experiment $(p<0.005)$. MT decreased when the forearm was obstructed only for the reverse experiment $(p<0.005)$. There was no significant effect of removing visual guidance on $\mathrm{MT}(p>0.05)$.

Passive responses of forearm. The limb's response to unexpected displacement when subjects maintained a half-pronated posture of the forelimb is illustrated in Figure 8. Relative static error was unaltered by changes 
in the amplitude of the displacement. Note that the summed relative values of the passive end point error were very close to zero (Fig. 8, left panel). There was, nevertheless, considerable variability in the passive end point error. This is indicated by the approximately $15 \%$ error, for all movement sizes, when the absolute value of end point error was evaluated (Fig. 8, right panel).

\section{Discussion}

Several recent reports concerned with the programming of arm movements in humans and monkeys (Polit and Bizzi, 1979; Kelso and Holt, 1980; Schmidt and McGown, 1980) have indicated that transient modifications in the physical constraints encountered during movement do not impair the accuracy of limb positioning. In agreement with the results of these earlier experiments, we have found that movements of magnitude similar to those studied by other investigators $\left(30^{\circ}\right.$ in our experiments, $20^{\circ}$ to $50^{\circ}$ in other reports) were relatively unimpaired by kinesthetic disturbances. In contrast, we found that accuracy of small movements was greatly impaired by perturbations of the arm occurring during

TABLE III

Movement time $e^{\alpha}$ grouped according to direction and size of movement

\begin{tabular}{cccccc}
\hline \multirow{2}{*}{ Movement } & \multicolumn{2}{c}{ Supination } & & \multicolumn{2}{c}{ Pronation } \\
\cline { 2 - 3 } \cline { 5 - 6 } & No-Stop & Stop & & No-Stop & Stop \\
\hline $3^{\circ}$ & 192.5 & 178.5 & & 200.8 & 1779.2 \\
$10^{\circ}$ & 199.6 & 188.4 & & 204.0 & 192.5 \\
$30^{\circ}$ & 209.2 & 200.7 & & 215.3 & 214.0 \\
\hline & Non-Reverse & Reverse & Non-Reverse & Reverse \\
\hline $3^{\circ}$ & 181.7 & 168.0 & & 189.3 & 168.7 \\
$10^{\circ}$ & 198.5 & 182.3 & & 196.7 & 183.8 \\
$30^{\circ}$ & 214.5 & 195.5 & 219.4 & 208.0 \\
\hline
\end{tabular}

${ }^{a}$ Movement time was measured in milliseconds.

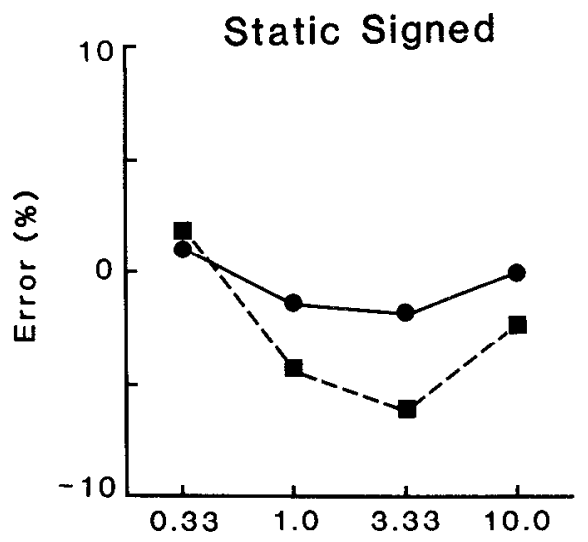

movement. Thus, the size of movement is a critical variable in determining whether the central signal to the muscles is adequate to specify the end point for the limb.

The observed differences in the consequences of perturbations during large and small movements raise questions as to the physiological mechanisms underlying the differences. There is substantial evidence that sensory receptors and motor units have their greatest sensitivity or dynamic range to small changes in input signals (Matthews and Stein, 1969; Tanji and Kato, 1973; Vallbo, 1973; Joyce et al., 1974; Freund et al., 1975; Hasan and Houk, 1975; Knibestöl, 1975; Marsden et al., 1978; Cooker et al., 1980; Hoffer and Andreassen, 1981). Since sensory receptors and the motor apparatus are most responsive to small input signals, it might have been expected that compensatory responses to peripheral disturbances would be best during precise or small movements. However, results from behavioral studies suggest the opposite since, for example, the precise temporal patterning of EMG activity seen during locomotion is dramatically altered by peripheral disturbances (Forssberg, 1979; Wand et al., 1980) and the finer aspects of position sense, sense of effort, and reproduction of some fine movements are severely impaired by disruption or removal of afferent input (Laszlo and Bairstow, 1971; Goodwin et al., 1972; Gandevia and McCloskey, 1977a, b).

An explanation of the observed greater disruption of precise or small movements by peripheral disturbances may be found by considering the interactions between central signals and sensory inputs at the $\alpha$ motoneuron when subjects attempt large and small movements. A central signal for large and rapid movements (as was the case here) would activate a large proportion of the motoneuronal pool, evident by the substantial EMG activity accompanying large movements, possibly saturating a large number of the individual motor units. This would tend to reduce the possibility that peripheral disturbances that excite muscle receptors, injected close upon

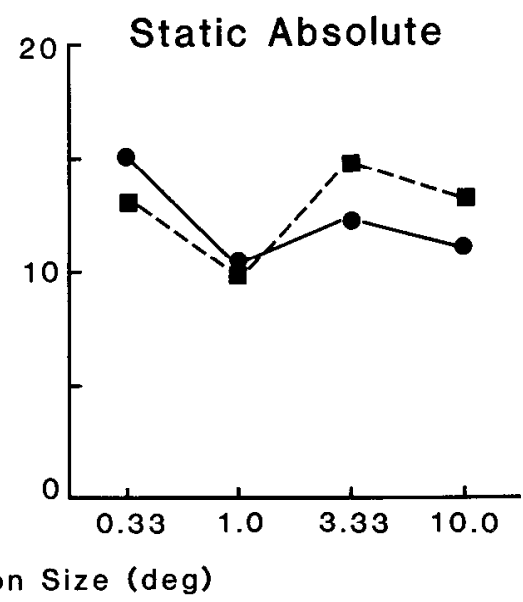

Figure 8. Passive responses of forearm. Mean error relative to displacement size for the group of subjects. Left, End point error expressed in terms of the mean actual difference between handle and original position measured when the forearm had settled. Displacement size did not affect static error. Right, Relative absolute static error was determined by summing the absolute values of the difference between handle and original position when the limb had settled. Notice that the variation in absolute static error is considerable (cf. comparable values on the left with those on the right) but displacement magnitude did not alter the amount of absolute end point error. 
the voluntary movement, would substantially increase muscle activity. In contrast, a central signal for small movements would activate only a modest proportion of the motoneuronal pool, thereby allowing an opportunity for substantial modulation of EMG activity by sensory inputs. There are parallels between this scheme for the importance of reflex responses in selectively affecting small movements and earlier observations indicating that reflex EMG only influenced a limb's response to small passive displacements. Joyce et al. (1974) studied the transfer of energy between a human's arm and a mechanical device that passively oscillated the arm. The oscillations always evoked reflex muscle responses, but the forces generated reflexively were only sufficient to cause negative work transfer (i.e., from the arm to the device) when the passive oscillations were small. It was suggested that during large passive forearm oscillations the inherent mechanical properties of the arm negated forces generated by muscle activity. Similarly, for large active movements, mechanical factors, such as limb inertia and viscoelastic properties of the muscle, might overwhelm any reflex responses generated by obstructions encountered during the movement (Bizzi et al., 1976). Despite the role of viscoelasticity and inertia in trajectories following perturbations, our second experiment suggests that a neurally mediated muscle response underlies the relatively greater static errors with perturbations of small movements. Changes in the size of the passive displacement delivered during postural stability did not modify end point error of the limb's free response, suggesting that mechanical factors contribute proportionately equal amounts of restorative force to a limb that is perturbed in varying amounts. By inference, a nonmechanical, possibly neural process was important in causing substantially greater static error for small voluntary movements that were perturbed.

An additional observation in human subjects that supports the notion that sensory inputs would be more disruptive for fine motor acts is an enhanced EMG responsiveness to kinesthetic inputs when these inputs are delivered as subjects attempt to make small changes in the activity of single motor units (Sanes and Evarts, 1983). It was found that passively produced changes in muscle length disrupted the ability of human subjects to generate a single impulse in a motor unit being studied, though the same stimulus had very little effect on the ability of subjects to maintain a steady, and low, discharge rate of the same motor unit. There is also a parallel between our results in human subjects and the results of Fromm and Evarts $(1977,1978)$ on pyramidal tract neurons (PTNs) in the motor cortex of the monkey. Identical kinesthetic stimuli occurring in association with performance of large and small movements had very different effects, with the stimuli causing intense PTN discharge during small movements but having relatively little effect during large movements. In these same studies, PTNs showed enhanced responsiveness to stimuli delivered during small movements as compared to immobility.

It has been proposed that regulation of muscle stiffness and inherent viscoelastic properties of a limb account for the trajectory and end point control of movements and for a large proportion of the mechanical response to perturbations occurring in the course of movement, with only a small residual part of the response being the result of a reflex neuromuscular response to the disturbance (Rack and Westbury, 1969; Bizzi et al., 1976; Abend et al., 1982). Our observation of the increased relative dynamic overshoot following perturbations of small as compared to large movements points to a progressively greater reflex contribution as movements become progressively smaller. In considering this dynamic overshoot, it may be noted that dynamic overshoot in a secondorder system following a step input is largely a function of the damping factor of the mechanical system. That is, the greater the damping factor, the less the dynamic overshoot. In addition, and most germane to the present discussion, when the damping factor is invariant, dynamic overshoot is a constant proportion of the input step function. If we assume that in our studies the damping factor of the forearm was constant, then the absolute dynamic overshoot for the range of movements studied in our experiments should have been scaled according to the ratio of movement sizes (i.e., 3:10:30). Stated in another way, the dynamic overshoot should have been the same proportion of the movement amplitude for all magnitudes of forearm rotation. But clearly (Figs. 3 and 4), proportionate dynamic overshoot increased linearly with decreases in movement size.

With regard to static error, the equilibrium point of a spring is unchanged if a mechanical disturbance is delivered to the spring for a finite time, provided the disturbance does not change the inherent mechanical properties of the system. Thus, if limb positioning is similar to the properties of linear springs, the static error should be invariant, independent of the presence or absence of a brief positional disturbance and of the size of the movement. Our results for small movements clearly failed to follow this model. There is additional recent evidence that argues against an open loop spring model hypothesis for end point accuracy. Day and Marsden (1982) trained humans to move against a reference viscous friction and then varied the viscous friction that opposed dynamic changes in limb position. If an unpredictable viscous load was presented at movement onset, the open loop spring model hypothesis would predict that final positional accuracy of the limb would be unaffected. The results indicated that unpredictable viscous loads did not disrupt end point control if the sensory apparatus of the interphalangeal joint of the thumb was intact, but that consistent overshoots and undershoots in end point positioning were observed when greater or less viscous loads, respectively, were unpredictably presented while the cutaneous and joint afferents were inactivated by anesthesia.

A significant difference between our study and others concerned with control of limb positioning was the use of intact subjects rather than deafferented preparations (Polit and Bizzi, 1979; Kelso and Holt, 1980). While current methods of deafferentation surgically remove or render functionally inactive most of the peripheral return from muscles, joints, and skin, the adequacy of these techniques in "deafferenting" subjects is questionable. Surgical deafferentation typically involves dorsal rhizo- 
tomy and leaves the ventral root efferents intact. The function of ventral root afferents in kinesthesia is unknown, but these neurons clearly mediate some deep mechanical sensations (Clifton et al., 1976); sensations that have been thought to be involved in position sense. An additional problem with the technique of ischemic deafferentation is that muscle weakness usually accompanies the inactivation of sensory afferents (Kelso and Stelmach, 1974).

Although it is true that available techniques for deafferentation fail to eliminate motor functions that would appear to require the sense of kinesthesia, it is not necessarily correct to interpret residual motor functions as evidence of the unimportance of peripheral input in regulating end point control. According to the open loop spring model hypothesis, the basis for the retained function following deafferentation has been attributed to the extraordinary efficiency and accuracy of central motor commands. The present experiments cast some doubt on this notion since we have demonstrated a mismatch between intended and actual final position when peripheral receptors of intact humans are transiently stimulated during small active movements. A possible explanation for the retained accuracy of motor performance following either acute or chronic deafferentation is that subjects rapidly adapt to the lack of peripheral information. The adaptive mechanism would necessarily be modification of the motor commands to the muscle and would be contingent on knowledge of results of each movement. Thus, if deafferented subjects received some sensory information (visual or auditory) about accuracy, then subsequent motor commands could be modified to improve future movements.

The present results bear on the psychophysical relationships among MT, required movement accuracy, and movement size that was initially described by Fitts (1954). Fitts noted that a movement's index of difficulty, defined by the movement amplitude and size of a target zone, would determine MT provided accuracy was held constant. A recent extension of Fitts' (1954) findings demonstrated that when MT was fixed, the error rate would be determined by the size of the movement and target (Schmidt et al., 1979). In the present experiments the index of difficulty for all movement sizes was identical since decreases in the movement size was accompanied by commensurate decreases in the target zone. If our data fit into the behavioral models derived from Fitts (1954), the observation that MT decreased (significantly only in the reverse experiment) as movement size diminished should have (and did) resulted in increases in error for faster movements. Similarly, since MT also decreased for all movement sizes following perturbations, it might have been expected that error would have increased for all movement amplitudes; but as the results showed, this expectation was realized only for the smaller movements. Most investigations concerned with the predictions of Fitts' (1954) model have dealt with movements considerably different from the rotary movements we used. In earlier experiments subjects typically moved a stylus between two target plates. This task is best suited to analyze trajectory (dynamic) errors, but is grossly inadequate to study end point error.

\section{References}

Abend, W., E. Bizzi, and P. Morrasso (1982) Human arm trajectory formation. Brain 105: 331-348.

Bernstein, N. (1967) The Co-ordination and Regulation of Movements, Pergamon Press, Oxford.

Bizzi, E., A. Polit, and P. Morasso (1976) Mechanisms underlying achievement of final head position. J. Neurophysiol. 39: 435-444.

Bizzi, E., P. Dev, P. Morasso, and A. Polit (1978) The effect of load disturbances during centrally initiated movement. J. Neurophysiol. 41: 542-556.

Clifton, G. L., R. E. Coggeshall, W. H. Vance, and W. D. Willis (1976) Receptive fields of unmyelinated ventral root afferent fibres in the cat. J. Physiol. (Lond.) 256: 573-600.

Cooker, H. S., C. R. Larson, and E. S. Luschei (1980) Evidence that the human jaw stretch increases the resistance of the mandible to small displacements. J. Physiol. (Lond.) 308: 6178.

Day, B. L., and C. D. Marsden (1982) Accurate repositioning of the human thumb against unpredictable dynamic loads is dependent upon peripheral feed-back. J. Physiol. (Lond.) 327: 393-407.

Fitts, P. M. (1954) The information capacity of the human motor system in controlling the amplitude of movement. J. Exp. Psychol. 47: 381-391.

Forssberg, H. (1979) Stumbling corrective reaction: A phasedependent compensatory reaction during locomotion. J. Neurophysiol. 42: 936-952.

Freund, H. -J., H. J. Büdingen, and V. Dietz (1975) Activity of single motor units from human forearm muscles during voluntary isometric contractions. J. Neurophysiol. 38: 933-946.

Fromm, C., and E. V. Evarts (1977) Relation of motor cortex neurons to precisely controlled and ballistic movements. Neurosci. Lett. 5: 259-265.

Fromm, C., and E. V. Evarts (1978) Motor cortex responses to kinesthetic inputs during postural stability, precise fine movement and ballistic movement in the conscious monkey. In Active Touch: The Mechanism of Recognition of Objects by Manipulation, G. Gordon, ed., pp. 105-117, Pergamon Press, Oxford.

Fukushima, K., K. Taniguchi, Y. Kamishima, and M. Kato (1976) Peripheral factors contributing to the volitional control of firing rates of the human motor units. Neurosci. Lett. 3: 33-36.

Gandevia, S. C., and D. I. McCloskey (1977a) Sensations of heaviness. Brain 100: 345-354.

Gandevia, S. C., and D. I. McCloskey (1977b) Effects of related sensory inputs on motor performances in man studied through changes in perceived heaviness. J. Physiol. (Lond.) 272: 653-672.

Goodwin, G. M., D. I. McCloskey, and P. B. C. Matthews (1972) The contribution of muscle afferents to kinaesthesia shown by vibration induced illusions of movement and by the effects of paralysing joint afferents. Brain 95: 705-748.

Goodwin, G. M., D. Hoffman, and E. S. Luschei (1978) The strength of the reflex response to sinusoidal stretch of monkey jaw closing muscles during voluntary contraction. J. Physiol. (Lond.) 279: 81-111.

Hasan, Z., and J. C. Houk (1975) The transition in the sensitivity of spindle receptors that occurs when the muscle is stretched more than a fraction of a millimeter. J. Neurophysiol. 38: 673-689.

Hoffer, J. A., and S. Andreassen (1981) Regulation of soleus muscle stiffness in premammillary cats: Intrinsic and reflex components. J. Neurophysiol. 45: 267-285.

Joyce, G. C., P. M. H. Rack, and H. F. Ross (1974) The forces 
generated at the human elbow joint in response to imposed sinusoidal movements of the forearm. J. Physiol. (Lond.) 240: 351-374.

Kelso, J. A. S., and K. G. Holt (1980) Exploring a vibratory system analysis of human movement production. J. Neurophysiol. 43: 1183-1196.

Kelso, J. A. S., and G. E. Stelmach (1974) Behavioral and neurological parameters of the nerve compression block. J. Motor Behav. 6: 179-190.

Knibestöl, M. (1975) Stimulus-responsive functions of slowly adapting mechanoreceptors in the human glabrous skin area. J. Physiol. (Lond.) 245: 63-80.

Knibestöl, M., and A. B. Vallbo (1980) Intensity of sensation related to activity of slowly adapting mechanoreceptive units in the human hand. J. Physiol. (Lond.) 300: 251-267.

Lashley, K. S. (1917) The accuracy of movement in the absence of excitation from the moving organ. Am. J. Physiol. 43: 169194.

Laszlo, J. I., and P. J. Bairstow (1971) Accuracy of movement, peripheral feedback and efference copy. J. Motor Behav. 3: 241-252.

Marsden, C. D., P. A. Merton, H. B. Morton, J. E. R. Adam, and M. Hallett (1978) Automatic and voluntary responses to muscle stretch in man. Prog. Clin. Neurophysiol. 4: 167-177.

Matthews, P. B. C., and R. B. Stein (1969) The sensitivity of muscle spindle afferents to small sinusoidal changes of length. J. Physiol. (Lond.) 200: 723-743.

Polit, A., and E. Bizzi (1979) Characteristics of motor programs underlying arm movements in monkeys. J. Neurophysiol. 42: $183-194$.

Poppele, R., and R. J. Bowman (1970) Quantitative description of linear behavior of mammalian muscle spindles. J. Neurophysiol. 33: 59-72
Rack, P. M. H., and D. R. Westbury (1969) The effects of length and stimulus rate on tension in the isometric cat soleus muscle. J. Physiol. (Lond.) 204: 443-460.

Sanes, J. N., and E. V. Evarts (1983) The regulatory role of proprioceptive input in motor control of phasic or maintained voluntary contractions in man. In Brain and Spinal Mechanisms of Movement Control in Man. New Developments and Clinical Applications, J. E. Desmedt, ed., Raven Press, New York, in press.

Schmidt, R. A., and C. McGown (1980) Terminal accuracy of unexpectedly loaded rapid movements: Evidence for a massspring mechanism in programming. J. Motor Behav. 12: 149161.

Schmidt, R. A., H. Zelaznik, B. Hawkins, J. S. Frank, and J. T. Quinn (1979) Motor-output variability: A theory for the accuracy of rapid motor acts. Psychol. Rev. 86: 415-451.

Soechting, J. F., and F. Lacquaniti (1981) Invariant characteristics of a pointing movement in man. J. Neurosci. 1: 710720.

Tanji, J., and M. Kato (1973) Firing rate of individual motor units in voluntary contraction of abductor digiti minimi muscle in man. Exp. Neurol. 40: 771-783.

Terzuolo, C. A., and P. Vivani (1980) Determinants and characteristics of motor patterns used for typing. Neuroscience 5 : 1085-1103.

Vallbo, A. B. (1973) Muscle spindle afferent discharge from resting and contracting muscles in normal human subjects. In New Developments in Electromyography and Clinical Neurophysiology, J. E. Desmedt, ed., Vol. 3, pp. 251-262, S. Karger, AG, Rasel.

Wand, P., A. Prochazka, and K. -H. Sontag (1980) Neuromuscular responses to gait perturbations in freely moving cats. Exp. Brain Res. 38: 109-114. 